\title{
Una visión histórica del programa de formación en enfermería perinatal en Colombia 1980 a 1990 '
}

\author{
Luz Magdalena González Poveda² \\ Yaneth Mercedes Parrado 3
}

doi:10.11144/Javeriana.ie19-1.vhpf

Cómo citar: Gonzales Poveda LM, Parrado YM. Una visión histórica del programa de formación en enfermería perinatal en Colombia 1980 a 1990. Investig Enferm. Imagen Desarr. 2017;19(1): 33-44. http://dx.doi.org/10.11144/Javeriana.ie19-1.vhpf

1. Articulo derivado de Investigación. Fecha de recepción: 24 de marzo de 2015. Fecha de aprobación: 15 de febrero de 2016.

2. Magíster en Enfermería con énfasis en el cuidado materno perinatal. Coordinadora de la Dimensión de Sexualidad, Derechos Sexuales y Derechos Reproductivos de la Secretaria de Salud de Cundinamarca. Correo electrónico: 1mgonzalezpo@unal.edu.co.

3. Doctora en Enfermería. Profesora asociada, Facultad de Enfermería. Universidad Nacional de Colombia. Correo electrónico: ymparradol@unal.edu.co 


\section{Resumen}

Objetivo: Describir y analizar el surgimiento y desarrollo del programa de Especialización de Enfermería Perinatal en la Universidad Nacional de Colombia, entre 1980 - 1990, a la luz del contexto nacional en educación, salud y enfermería. Método: Investigación cualitativa con fuentes de información documental y entrevistas testimoniales de actores directos que permitieron la reconstrucción del hecho histórico. Resultados: El surgimiento de la Especialización de Enfermería Perinatal en la Universidad Nacional de Colombia fue directamente influenciado por politicas y estrategias de desarrollo del ámbito nacional e internacional que constituyeron un eje central en el planteamiento y posterior desarrollo de la especialización. Por otra parte, los procesos internos de la Universidad Nacional y en especial en la Facultad de Enfermería en temas relacionados con la reforma de la educación superior y el fortalecimiento de los posgrados en enfermería a nivel latinoamericano, fueron hechos claves para el alcance del programa. Finalmente, el liderazgo y experiencia de las docentes, junto con las asesorias internacionales, concretaron la idea de la Especialización en Enfermería Perinatal para plasmarla en un currículo que lograra formar recurso humano capaz de dar respuesta de manera efectiva a las necesidades en salud de las mujeres y los recién nacidos. Conclusiones: El surgimiento de la Especialización en Enfermería Perinatal en la Universidad Nacional de Colombia marcó una transformación en el modelo de atención de la madre y el neonato, con implicaciones en la práctica, la investigación y las políticas de atención emanadas desde el Ministerio de Salud.

Palabras clave: enfermería neonatal; historia de la enfermería; educación en salud

\section{A historical overview of the training program in perinatal nursing in Colombia from 1980 to 1990}

\section{Abstract}

Objective: To describe and analyze the emergence and development program of Specialization in Perinatal Nursing at the National University of Colombia, between 1980 and 1990, in the light of the national context in education, health and nursing. Method: Qualitative research with sources of information and witness interviews to direct actors that allowed the reconstruction of the historical fact. Results: The emergence of the Specialization in Perinatal Nursing at the National University of Colombia was directly influenced by policies and development strategies of national and international level constituted as a central axis in the planning and further development of the specialization. Moreover, the internal processes of the National University and especially in the School of Nursing on issues related to the reform of higher education and strengthening of graduate programs in nursing in Latin America were key issues in the scope of the program. Finally, leadership and experience of teachers, along with international consultants, materialized the idea of the Specialization in Perinatal Nursing to be brought into a curriculum that managed to train human resources capable of responding effectively to the health needs of women and newborns. Conclusions: The emergence of the Specialization in Perinatal Nursing at the National University of Colombia marked a transformation in the model of care for the mother and newborn, with implications for practice, research and care policies emanating from the Ministry of Health. 


\section{Uma visão histórica do programa de formação em enfermagem perinatal na Colômbia 1980 a 1990}

\section{Resumo}

Objetivo: Descrever e analisar o surgimento e desenvolvimento do programa de Especialização de Enfermagem Perinatal na Universidade Nacional da Colômbia, entre 1980 - 1990, à luz do contexto nacional em ensino, saúde e enfermagem. Método: Pesquisa qualitativa com fontes de informação documental e entrevistas testemunhais de atores diretos que permitiram a reconstrução do fato histórico. Resultados: O surgimento da Especialização de Enfermagem Perinatal na Universidade Nacional da Colômbia foi diretamente influenciado por políticas e estratégias de desenvolvimento do âmbito nacional e internacional que constituíram um eixo central no esboço e posterior desenvolvimento da especialização. Por outra parte, os processos internos da Universidade Nacional e em especial na Faculdade de Enfermagem em temas relacionados com a reforma do ensino superior e o fortalecimento dos programas de pós-graduação em enfermagem no nível latino-americano, foram fatos chave para o escopo do curso. Finalmente, a liderança e experiência das docentes, junto com as consultorias internacionais, concretaram a ideia da Especialização em Enfermagem Perinatal para materializá-la em um currículo que conseguisse formar recurso humano capaz de responder efetivamente às necessidades em saúde das mulheres e os recémnascidos. Conclusões: O surgimento da Especialização em Enfermagem Perinatal na Universidade Nacional da Colômbia marcou uma transformação no modelo de atendimento da mãe e o neonato, com implicações na prática, a pesquisa e as políticas de atendimento emanadas desde o Ministério da Saúde.

Palauras-chave: enfermagem neonatal; história da enfermagem; ensino em saúde 


\section{Introducción}

Durante la segunda mitad el siglo XX, las altas tasas de morbi-mortalidad materna y perinatal, se convirtieron en una de las características del perfil epidemiológico en los países latinoamericanos, relacionadas con una precaria cobertura de los servicios de salud materno infantil, escasez de recurso humano capacitado en el área, ausencia de programas efectivos que atendieran las poblaciones más vulnerables y existencia de barreras culturales, geográficas y económicas que impedían el acceso adecuado de la población a los servicios de salud.

En los años setenta se generaron movimientos políticos en el ámbito internacional, regional y nacional, que pretendian fomentar el desarrollo de la población por medio de cambios estructurales en aspectos socio-económicos que involucraban a diferentes sectores del estado, entre ellos; salud y educación.

Bajo estas circunstancias, y como una estrategia para dar respuestas a las necesidades de la población, se fortalecieron los convenios de cooperación internacional, los programas de salud pública con orientación de Atención Primaria en Salud (APS) y la cualificación del recurso humano.

En Colombia, para el desarrollo de estas politicas fueron actores estratégicos: el Ministerio de Salud, las asociaciones científicas y las universidades. Desde la academia se planteó la necesidad de cualificar el recurso, expandir el conocimiento y abrir campo en la investigación, para dar respuesta a los problemas del momento. La puesta en marcha del fortalecimiento del programa en salud materno-infantil y el marco de reglamentación de la Ley 80 de 1980 (por el cual se organiza el sistema de educación post-secundaria y cuyo Artículo 37, establece la especialización como formación avanzada), constituyeron referentes para el surgimiento de la Especialización de Enfermería Perinatal en la Universidad Nacional de Colombia (1).

Como respuesta a estos eventos, la Facultad de Enfermería de la Universidad Nacional propuso la creación e implementación de la especialización, lo cual constituyó un hecho importante para la atención de la madre y el recién nacido, ya que permitió que desde el desarrollo del programa y el posterior desempeño de las egresadas, se plantearan cambios estructurales y metodológicos en las politicas de atención de la madre y el perinato, enfocadas a la disminución de los altos índices de enfermedad y muerte.

En la revisión documental de fuentes primarias, literatura científica, y conversatorios preliminares con docentes pioneras en el área, no se encontró un análisis del origen, la estructura del plan de estudios, la relación profesoras- estudiantes, la historia, entre otros aspectos explorados que se relacionan con el inicio del programa de Especialización de Enfermería Perinatal en la Universidad Nacional de Colombia en el periodo comprendido entre 1980 y 1990.

Partiendo de este marco, la presente investigación responde a los siguientes interrogatorios: ¿Cuáles fueron los aspectos del contexto que influyeron en el desarrollo de la Especialización de Enfermería Perinatal en la Universidad Nacional de Colombia en el periodo comprendido entre 1980 y 1990?, ¿Cómo nació la Especialización en Enfermería Perinatal en la 
Universidad Nacional de Colombia? y ¿Qué aspectos conceptuales, teóricos y de la práctica se identificaron en los elementos de la estructura curricular (profesoras, estudiantes y plan de estudios) de la Especialización en Enfermería Perinatal en la Universidad Nacional de Colombia en el periodo comprendido entre 1980 y 1990 ?

Esta investigación constituye un referente nacional que incursiona en la investigación de corte histórico en enfermería perinatal y aporta, desde una perspectiva analítica, conocimiento necesario para recuperar la memoria histórica y fortalecer el cuerpo teórico de la profesión y de la especialización.

Desde el punto de vista social, generar investigación histórica en enfermería contribuye a la identidad profesional de las enfermeras. Conocer las circunstancias que rodearon los hechos históricos, facilita la comprensión de las experiencias durante la formación, el ejercicio profesional, los roles adquiridos, la autonomía y el liderazgo alcanzado. Se espera aportar con un marco de referencia clave para contrastar, reorientar y cualificar la enfermería perinatal a partir de las tendencias actuales y las proyecciones en investigación, práctica y formación.

Así mismo y teniendo en cuenta lo expresado por Durán de Villalobos: "la mayoría de la investigación histórica de enfermería actual está basada más en las personas y no hay mucho examen de patrones de la práctica, modelos, cambios en los procedimientos y otros focos de interés" (2). Esta investigación pretendió aportar, más allá de las historias de vida, a los procesos educativos y de salud que tuvieron como resultado final un impacto en los patrones de la práctica en el área de enfermería materno perinatal.

El objetivo general de este estudio fue describir y analizar cómo fue el surgimiento y desarrollo del programa de Especialización de Enfermería Perinatal en la Universidad Nacional de Colombia, en el periodo comprendido entre 1980- 1990, con el fin de aportar hechos históricos y un análisis contextual y curricular, que contribuya a la formación del recurso humano, al fortalecimiento y la historia de la disciplina y la profesión de enfermería.

\section{Método}

Investigación cualitativa desarrollada con base en la historia institucional del programa de formación de especialistas en enfermería perinatal, las dinámicas del pasado, el surgimiento, el desarrollo curricular y las relaciones existentes entre las politicas de salud regionales y nacionales en torno al programa en mención. La unidad de estudio se consideró cronológica, enfocada en el análisis de los hechos históricos del periodo comprendido entre 1980 y 1990. Las fuentes utilizadas fueron de dos tipos: documentales; consideradas elementos básicos para la consolidación de la información insumo para el análisis y testimoniales; que permitieron plasmar la experiencia y vivencia de las personas para contrastar o complementar los hallazgos.

Mediante la revisión de archivos de la Universidad en sus cuerpos colegiados: Consejo Superior Universitario (CSU), Consejo Académico (CA), Consejo Directivo de la Facultad y Comité Asesor del programa, se obtuvo la mayor parte del material analizado. 
La consulta en bibliotecas, bases de datos, motores de búsqueda, periódicos, revistas y el análisis de documentos e informes emanados desde el Ministerio de Salud, relacionados con políticas de atención en el área materno perinatal, permitió la construcción del entramado del contexto.

Se consideraron fuentes primarias: documentos escritos durante el periodo de estudio o previos a él, que provenían de actores institucionales, nacionales o internacionales y registros de actas que dan testimonio de los procesos que se dieron durante el surgimiento del programa. También entrevistas testimoniales a profesoras y exalumnas, personas pioneras o líderes del proceso, que dieron cuenta de hechos relacionados con la práctica, la investigación y la docencia del programa de formación de la especialización.

Las fuentes secundarias fueron conformadas por material relacionado con la dinámica en salud y educación de la época. Se incluyeron artículos de revistas, evaluaciones e informes. Esta información y datos fueron recopilados en bibliotecas, búsquedas en línea, artículos, informes, centros de documentación y archivos del programa, entre otros.

En el procedimiento metodológico se identificaron cuatro etapas que permitieron llevar un orden en el trabajo, más no establecer una estructura lineal, pues los momentos se superponen para lograr conocer los hechos particulares en la Facultad de Enfermería.

El aspecto ético se basó en los principios de honradez intelectual y derechos de autor para el manejo de fuentes primarias y secundarias, y en el consentimiento informado para la presentación de los testimonios. Además se contemplaron los lineamientos dados por la Resolución 8430 del 4 de octubre de 1993 "por la cual se establecen normas científicas, técnicas y administrativas para la investigación en salud” en la República de Colombia (3).

\section{Resultados}

Los resultados obtenidos se agruparon en tres categorías o capítulos desarrollados a continuación en lo que se muestra la influencia de las politicas, el surgimiento y desarrollo curricular del programa.

\section{Una especialización marcada por las políticas de salud y desarrollo de la profesión.}

La Especialización en Enfermería Perinatal en la Universidad Nacional de Colombia, como una nueva propuesta curricular, se vio influenciada por la ocurrencia de diferentes sucesos en los escenarios nacionales e internacionales dentro de los cuales se pueden identificar las nuevas políticas en el ámbito de salud y educación y el sello particular en la perinatología en Latinoamérica. La creación del Centro de Perinatología y Desarrollo Humano (CLAP), hacia 1970 estableció las directrices para la atención del binomio madre e hijo. Este Centro, con el apoyo de entidades como la Organización Panamericana de la Salud (OPS), se encargaron de brindar un soporte irrestricto al desarrollo de 
estrategias que propendieron por la disminución de los indices de mortalidad materna y perinatal en cada país de América Latina (4).

La formación del recurso humano en perinatologia, el apoyo y acompañamiento en la investigación, la cooperación técnica en relación con los lineamientos para la formulación del programa y la difusión respecto a la posibilidad de acceso a la bibliografía relacionada con la estrategia perinatal (4) fueron los aportes del CLAP para la creación del programa.

De otro lado, las políticas en salud destacan la influencia de los planes que tomaron como referente la atención materno infantil en Colombia, entre los que tenemos: El Plan Decenal de Salud Pública (PDSP) para el periodo 1968-1977 (5); el Plan Hacia la Equidad en Salud 1982-1986 (6) y el Plan Nacional para la Supervivencia y el Desarrollo Infantil 1986 - 1989 (7). Los cuales en conjunto buscaron mejorar el nivel de salud de la población materno infantil mediante la capacitación del recurso humano en salud, la ampliación de coberturas de atención, la captación precoz de la gestante, así como el control de la natalidad y la cooperación docente asistencial. Por último, facilitaron la clasificación del riesgo perinatal, lo cual permitió brindar atención adecuada por niveles de complejidad.

Entre tanto, en el sector educativo se replanteaba la cualificación de la educación por medio del Decreto 80 de enero 22 de 1980, del Ministerio de Educación Nacional de Colombia, estatuto orgánico de la educación superior en el país. Este apuntó principalmente a definir los niveles de educación superior, asignarle funciones, organizar, delimitar las modalidades y redefinir los objetivos del sistema de educación superior, con el fin de superar los objetivos de docencia, y pasar al campo de producción de conocimientos a través del énfasis en actividades de investigación, al igual que la vinculación de la universidad con los procesos de desarrollo socioeconómico del país (1).

Con esta directriz los centros de educación superior propusieron programas de posgrado que apuntaran a contribuir en la solución de problemas prioritarios de la sociedad colombiana, en este caso, la salud de las mujeres y los niños. La Facultad de Enfermería presenta la propuesta de desarrollo de la Especialización en Enfermería Perinatal, hallazgo similar a lo reportado por Velandia A con respecto a las tendencias de educación para enfermería en la década de los 80 (8).

Sumado a estos aspectos del contexto, el desarrollo de la post-graduación en enfermería en Latinoamérica hacia la década de los ochentas y los profundos cambios curriculares en las escuelas de enfermería (9) marcaron una influencia importante en el surgimiento de la especialización.

Por medio de los documentos y testimonios recolectados se pudo evidenciar que efectivamente el programa recibió todos estos influjos y se nutrió de ellos para el propósito de "preparar recurso humano para la atención de enfermería a la unidad materno-fetal y el recién nacido, en los niveles de atención primaria, secundaria y terciaria, dentro de un criterio de integración de las etapas pre, trans y neonatal y en un sistema de atención regionalizado apoyado en el desarrollo de programas de investigación" (10). 


\section{Surgimiento del programa luego de muchos acuerdos de voluntades}

Al interior de la Universidad Nacional de Colombia (11) y en especial de la Facultad de Enfermeria, se comenzaron a dar procesos tendientes al fortalecimiento de la post-graduación, tales como la inclusión de esta dentro de los planes de desarrollo de la formación avanzada, la investigación y la capacitación del cuerpo docente (12). Con estas directrices se comenzó a gestar el programa hacia el año de 1982, a cargo del Departamento de Salud Pública y Materno infantil, en cabeza de la profesora Maruja Polania de Salazar, que según los testimonios de las profesoras, fue la ideóloga de este Programa (13).

Posterior a la formulación de la propuesta de la Especialización se nombró a la profesora Lucy Muñoz de Rodríguez como Directora Académica del mismo, de igual manera, se conformó el comité asesor integrado por docentes del área (12). A partir de ese momento se iniciaron contactos con OPS/ CLAP (12), quienes prestaron asesoria constante y brindaron capacitación en perinatología a docentes de la Facultad, profesionales del Ministerio de Salud y la Secretaria de Salud de Bogotá, quienes se desempeñaban en las áreas directivas de los programas de materno infantil (13).

Otro acompañamiento importante en la formulación del programa fue el dado por la Facultad de Medicina de la Universidad Nacional y del Instituto Materno Infantil de Bogotá (14).

La primera propuesta fue socializada en mayo de 1983 (12) ante el Consejo Directivo de la Facultad de Enfermería. Se presentaron aspectos relacionados con la estructuración, contenidos y requisitos para acceder al título de Especialista en Enfermería Perinatal en la Facultad de Enfermería de la Universidad Nacional de Colombia. Se contempló la especialización como un programa que:

Preparará al profesional de enfermería para promover la salud durante el proceso de la reproducción, prevenir los problemas que afectan la reproducción y asumir, con base científica las funciones de participación en los niveles de atención secundaria y terciaria; organizar y administrar servicios de enfermería perinatal y realizar y contribuir en las investigaciones perinatales con detección e identificación de problemáticas, sobre las cuales se propongan soluciones viables y se redefinan acciones de enfermería $(10,16)$.

Luego de algunos ajustes sugeridos por asesores externos y docentes de la Facultad (11), se presentó el documento final con revisiones y correcciones para obtener la aprobación del Consejo Directivo de la Facultad de Enfermería en octubre de 1983. Fue aceptado en esta instancia y enviado al Comité de Estudios de Posgrado quien lo analizó y recomendó en el Acta 037 del 31 de octubre de 1983, la creación del programa; esta decisión fue ratificada por el Consejo Académico el 2 de diciembre del mismo año.

El Consejo Superior Universitario aprobó el programa y sus objetivos de formación el día 12 de diciembre de 1983 mediante Acuerdo 166 de 1983 
Acta 54, en esta misma sesión autorizó al Consejo Académico para aprobar el plan de estudios (15). Finalmente, para dar comienzo al programa, el Comité de Posgrados de la Facultad planteó el calendario académico para iniciar el segundo semestre de 1984. El Consejo Directivo de la Facultad aprobó el plan calendario (12), el cual comenzaba con las inscripciones en junio de 1984 y finalizaba en diciembre 7.

El factor que más influyó en el surgimiento de la eespecialización, al interior de la Facultad de Enfermería, fue sin duda el empoderamiento y liderazgo de las profesoras del Departamento de Salud Pública y Maternoinfantil, quienes lograron que confluyeran actores de diversos estamentos nacionales e internacionales en la formulación del programa, hecho que le dio un gran soporte y proyección a la Especialización de Enfermería Perinatal.

\section{El currículo una propuesta novedosa.}

Paralelo a todo el proceso de surgimiento, aconteció la estructuración curricular y la preparación del cuerpo docente para asumir el reto de liderar la primera Especialización en Enfermería Perinatal del país

Dentro de la propuesta de contenidos programáticos presentada en 1983 (10), se contemplaron tres áreas en la malla curricular; la primera denominada: Proceso de Apoyo de Enfermeria, donde se agruparon todas aquellas asignaturas que le permitirian al estudiante profundizar en conocimientos y desarrollar las habilidades específicas para su desempeño en el campo de la atención prenatal, compuesta por las siguientes tres asignaturas: Seminario de Investigación en Enfermería y Monografía, Seminario de Epidemiologia y Seminario de Proceso de Enfermería.

La segunda área se denominaba: Fundamentación Científica Clínica y Comunitaria, su finalidad era profundizar en los principios biopsicosociales especializados para la atención de enfermeria integral a la madre y el niño en las etapas pre, trans y neonatal; incluyendo las fases preconcepcionales como precursoras del nuevo ser (10). Esta área estaba integrada por las asignaturas de Seminario de Fundamentación Biomédica, Seminario de Sociedad y Familia, y Seminario del Ciclo Vital de la Pareja.

El área tres denominada: Clínica Especializada de Enfermería Perinatal Seminario y Práctica, buscaba profundizar los conocimientos de enfermería y adquirir habilidades para la atención de integral de enfermería a las embarazadas y neonatos de alto, mediano y bajo riesgo, incluyendo componentes administrativos de docencia e investigación.

Las asignaturas de este bloque, Seminario y Práctica de Enfermería Perinatal I, Seminario y Práctica de Enfermería Perinatal II y Residencia de Enfermería Perinatal, tuvieron un componente práctico muy alto. En su parte teórica buscaban integrar los conocimientos adquiridos en los diferentes seminarios para lograr una atención integral de la mujer, la gestante y el recién nacido en situaciones de salud en riesgo (10). 
De las asignaturas teóricas la que más intensidad horaria tenía era el Seminario de Fundamentación Biomédica y en la parte práctica la asignatura Residencia de Enfermería Perinatal, esta experiencia le aportó a los estudiantes una visión global de la situación en salud de la mujer y sus implicaciones en el desarrollo de los procesos reproductivos.

Las prácticas se llevaron a cabo en centros hospitalarios de alto nivel como el Instituto Materno Infantil y el Hospital Lorencita Villegas de Santos. Lo correspondiente a control prenatal de bajo riesgo, se trabajó en Centros de salud del Distrito (17).

El pensum anteriormente descrito se desarrollaba en cuatro trimestres con una la intensidad horaria de 1700 horas; sin embargo, en julio de 1984 se realizó la primera restructuración al programa (18), quedando con una intensidad horaria de 1740, distribuida en tres semestres. Esta redistribución aumentó horas para las asignaturas de Monografía, Seminario de Proceso de Enfermería y Seminario de Epidemiologia, además de fusionar Enfermería Perinatal I y II (18). El último ajuste en horas para asignaturas sucedió en la segunda cohorte. Se disminuyeron horas especialmente en lo concerniente a la práctica clínica, pues se eliminó la Residencia de Enfermería Perinatal y se aumentaron horas a la parte práctica dirigida a la atención comunitaria y familiar según lo manifestaron egresadas del programa (14). El incremento de horas en estas asignaturas demuestra una clara intención de fortalecer e impulsar el desarrollo investigativo en el área comunitaria.

Se reconoce que este plan de estudios se articulaba con el objetivo de reducir la morbilidad materna perinatal planteado en las politicas de salud de la época. A pesar de que el énfasis de dichas políticas se centraba en el empoderamiento de lo comunitario, también se buscó lograr atención especializada según el riesgo perinatal detectado (19), esto se logró con un recurso humano en enfermería idóneo y cualificado, resultado de los elementos pedagógicos brindados durante el proceso de consolidación de la especialización.

Los planes de estudios manejados en las dos primeras cohortes mostraron una fuerte influencia biomédica; sin embargo, la directora del programa y las estudiantes de la época refieren que la incursión en el proceso de enfermería fue un insumo valioso para el tránsito de la atención de enfermería perinatal, al cuidado de la mujer gestante y su hijo por nacer.

Tal vez, el aporte más importante, fue introducir el paradigma de la perinatología para el área de enfermería el cual hace referencia a:

Un conjunto de conocimientos científicos y técnicos sobre la reproducción humana y sus efectos sobre la madre y el neonato. En su sentido estricto comprende el periodo de vida humana entre la vigésima semana de gestación y el vigésimo octavo día de vida extrauterina. Y en su sentido amplio todas las acciones tendientes a una reproducción feliz. La perinatología en su sentido esencial, se ubica como una especialidad relacionada con las etapas fetal y neonatal en forma integrada (10). 
Por otra parte, los docentes que desarrollaron la especialización en los años ochentas contaban con un amplia trayectoria en la atención materno infantil y en el Proceso de Atención de Enfermería (PAE). Se contó también con la participación de docentes de ciencias básicas y medicina, situación que se evidencia en la formulación de las asignaturas especialmente en el área de fundamentación biomédica.

El cuerpo docente se cualificó de acuerdo al Plan de Desarrollo de la Facultad (1982), donde se otorgó un capitulo especial a la formación avanzada en perinatología.

Desde lo investigativo, las docentes de la época lograron desarrollar múltiples investigaciones en temas relacionados con gestación y puérperas, parto y puerperio, neonatos y bases conceptuales de la especialización (20).

La Facultad de Enfermería y en especial el Departamento de Salud Pública y Materno Infantil hizo un importante despliegue de recursos en cuanto a cualificación del personal docente, con el fin de lograr implementar un programa de especialización de alta calidad que respondiera a las necesidades de formación de recurso humano en salud en el área de perinatología.

El profesorado fue uno de los puntos importantes en la creación y desarrollo de la especialidad, ya que sin el liderazgo de este grupo de enfermeras no se hubiera logrado el objetivo. Ellas refieren haber sido un grupo fuerte de apoyo y colaboración mutua (14).

En lo concerniente a los estudiantes, fueron en su mayoría enfermeras que se desempeñaban en el área docente. Otro grupo de estudiantes fueron las enfermeras que participaban en cargos directivos en la atención materno-infantil en las áreas locales y regionales. Ya como estudiantes activas del programa, resulta meritorio resaltar el liderazgo que ejercieron en los sitios de práctica donde se les involucraba desde la formación y se les daba la responsabilidad de liderar nuevos proyectos de atención perinatal dentro de las instituciones (17).

Como egresadas, la principal función fue implementar las directrices de la perinatología a nivel nacional, regional y local. Al respecto la Directora del Programa de ese entonces manifiesta: "todas nuestras egresadas fueron líderes en las áreas que se desempeñaron, sin embargo, ese hecho hizo que muchas de ellas se desenvolvieran en cargos administrativos y la parte de cuidado directo a las mujeres y sus recién nacidos no se dio como se hubiese querido" (13).

Otra función importante de las egresadas tuvo que ver con la participación en el desarrollo de políticas de atención materno infantil a nivel nacional y la creación de la Asociación Colombiana de Perinatología (ACOPEN), ente que direccionó la atención del binomio madre hijo hacia finales de 1980 y comienzos de 1990.

Es de resaltar que luego del surgimiento y posicionamiento de la especialización en los años ochentas, se logró por medio del plan de estudios, que los profesores y los estudiantes ganaran un espacio significativo en el 
país, en cuanto a preparación de recurso humano clínico y administrativo, investigación y asesoría en políticas de salud materno perinatal.

Todo este proceso de desarrollo conceptual y temático del programa logró su objetivo en cuanto al perfil de los egresados, ya que se buscaba preparar profesionales de enfermería capaces de desempeñar funciones administrativas, asistenciales, de docencia y de investigación en el campo de la atención perinatal; es decir, en los tres niveles de atención.

Los planteamientos de esta nueva concepción en la atención de la madre y el niño, concebidos desde la especialización, fueron la base fundamental para ganarse un merecido reconocimiento y liderazgo, que hoy permiten identificar la práctica de enfermería en esta área como:

El área de conocimiento que se encarga de promover el cuidado de madre, perinato y familia por medio de la interacción con la enfermera donde se comparten experiencias, habilidades y percepciones acerca del cuidado y se generan compromisos para mantener la salud y la calidad de vida bajo los principios de autonomía, dignificación del ser humano y respeto por las costumbres relacionadas con el ejercicio de la maternidad-paternidad (21).

Ahora bien, en contraste con la actualidad y la realidad del país frente a la situación materno perinatal y la necesidad de formación en esta área. Se resalta la importancia de incluir en los programas curriculares de pregrado y de posgrado, temáticas relacionadas con los hallazgos de la investigación histórica en enfermería. Este aspecto contribuye a fortalecer la identidad y retomar el liderazgo que la profesión esgrimió en otros momentos, afianzando el desempeño de enfermería en los grupos interdisciplinarios y su autonomía profesional.

Persiste la necesidad de que las enfermeras argumenten frente a instancias políticas y de salud los desarrollos que la profesión ha logrado en el área materno perinatal, con el fin de obtener una participación más relevante en los niveles decisorios. De la misma forma, se considera fundamental el reconocimiento de fenómenos de interés vigentes relacionados con los avances conceptuales, teóricos, tendencias en investigación y la práctica a la luz del contexto social, económico y educativo del país.

\section{Conclusiones}

Con la Especialización en Enfermería Perinatal se implementó el nuevo paradigma de atención materno infantil, identificado como Atención Perinatal, abordando aspectos de las políticas en educación y en salud que en la década de los ochenta direccionaron estas dos áreas de hondo interés social.

La investigación histórica en el área de Enfermería Perinatal evidencia la influencia del contexto en las prácticas de cuidado de la mujer y su hijo por nacer, y la formación de enfermeras especialistas en área perinatal en el periodo estudiado.

44 Los aportes del programa se pueden identificar en una atención con enfoque perinatal dirigida a la gestante, el neonato y la puérpera, articulada 
en los diferentes niveles de atención del sistema, utilizando principios de la estrategia de APS, sin desconocer el fuerte componente médico biológico y el escaso abordaje en la participación comunitaria.

La estructura del plan de estudios tuvo un marcado sello clínico, que se evidenció en la formación en Epidemiologia y Fundamentación biomédica, con una propuesta de residencia clinica; sin embargo, el desarrollo de asignaturas como Proceso de Enfermería y Seminario de Investigación que conducía a Monografía, permitieron a las especialistas incursionar en el manejo de los programas en las diferentes regiones de la geografía colombiana, en un nivel más complejo de formación y en la propuesta de temas de investigación más cercanos al conocimiento propio de la disciplina de enfermería.

\section{Financiación}

Ninguna

\section{Conflicto de interés}

Ninguno

\section{Referencias}

1. Parra R, Jaramillo B. La educación superior en Colombia. Cresalac -Unesco. Caracas- Venezuela: UNESCO. Julio de 1985.

2. Duran MM. Diseños de Investigación. Material preparado para la asignatura Seminario de Investigación I de la Maestría en Enfermería. Universidad Nacional de Colombia, Bogotá. 1990.

3. República de Colombia. Ministerio de Salud Pública, Resolución 008430 de octubre 4 de 1993, por la cual se establecen normas científicas, técnicas y administrativas para la investigación en salud. [Internet] [Consultado el 12 de febrero de 2012] Disponible en: http://www.dib. unal.edu.co/promocion/etica_res_8430_1993.pdf

4. Centro Latinoamericano de Perinatología y Desarrollo Humano (CLAP). Salud perinatal. Boletín del Centro Latinoamericano de Perinatología. Montevideo Uruguay. 1983; 11 (2)

5. Ministerio de Salud Pública. Dirección de Atención Médica. División Materno Infantil. Documento Mimeografiado Evaluación del programa materno Infantil del Ministerio de Salud Pública. Colombia, 1969- 1974.

6. Departamento Nacional de Planeación Ministerio de Salud. Cambio con equidad. Plan Nacional de Desarrollo. Colombia, 1983-1984.

7. Dirección de Atención Médica. División materno infantil y dinámica de población. Subprograma enfermedad perinatal. Plan nacional de la supervivencia de la infancia. Control de la mortalidad perinatal en Colombia. Grupo de Perinatología. Documento técnico, enero 14 de 1985.

8. Velandia AL. Tendencias de la educación de enfermería en Colombia en los últimos 25 años 1960 1985. Revista Investigación y Educación en Enfermería. 1998; VI (1) 
9. Verderese O. Análisis de la enfermería en la América Latina. Educación y salud. 1979; 13(4).

10. Facultad de Enfermería. Universidad Nacional de Colombia. Estudios de Posgrado. Programa de Especialización en Enfermera perinatal. Documento inédito. Bogotá, septiembre de 1983.

11. Consejo Académico. Universidad Nacional de Colombia. Libro de actas 1981. Acta 005 de junio 12 de 1981. p.5. Bogotá, Colombia.

12. Bogotá. Colombia. Consejo Directivo. Libros de Actas Facultad de Enfermería. Universidad Nacional de Colombia. 1978-1990.

13. Muñoz L. Entrevista concedida a Magdalena González. Mayo de 2011.

14. Ruiz CH. Entrevista concedida a Luz Magdalena González. Mayo de 2011.

15. Consejo Superior Universitario. Universidad Nacional de Colombia. Libro de Actas N Acta No 54. Acuerdo 166 del 12 de diciembre de 1983. Bogotá, Colombia.

16. Facultad de Enfermería. Universidad Nacional de Colombia. Estudios de Posgrado. Programa de Especialización en Enfermera perinatal, documento inédito. Bogotá Agosto de 1987.

17. Hernández LM. Entrevista Concedida a Luz Magdalena González. Mayo de 2011.

18. Consejo Directivo Facultad De Enfermería. Universidad Nacional de Colombia. Libro de actas, Acta 020. p. 4,5,6. año 1984. Bogotá, Colombia.

19. Ministerio de Salud. Taller sobres aspectos gerenciales en salud materna y perinatal. Cali, Colombia. Febrero de 1998

20. Bernal Roldán MC. Producción investigativa y proceso de reconceptualización del programa de especialización en enfermería perinatal. Revista Avances en Enfermería. 1995; XIII (2) julio - diciembre

21. Ruiz CH, Muñoz L. Cuidado de Enfermería materno perinatal, en su rol asistencial, gerencial, docente e investigativo. Cuidado y práctica de la enfermería. Facultad de Eenfermería. Universidad Nacional de Colombia, Bogotá. 2000. 